\title{
Phase Evolution Analysis During Real-Time Solid-State Chemical Lithiation of Crystalline Thin Window Silicon Membranes Using Low-Loss STEM-EELS Imaging
}

\author{
Vladimir Oleshko
}

NIST, Gaithersburg, Maryland, United States

Silicon is one of the most promising anode materials for high-energy-density Li-ion batteries with an exceptional capacity of $3579 \mathrm{mAh}^{-1}$ assuming $\mathrm{Li}_{15} \mathrm{Si}_{4}$ [1, 2]. Its practical use, however, is hindered by high volume expansion (up to $280 \%$ ) during Li insertion often resulted in contact loss with electrodes and rapid capacity fading. Various advanced designs of nanostructured Si-based composite anodes have been employed to demonstrate their high performance and better accommodate strain while allowing shorter diffusion lengths for $\mathrm{Li}^{+}$ions and faster charge/discharge rates. Meanwhile, a fundamental understanding of phase structures, compositions, and transformations during lithiation of Si and kinetic mechanisms is still lacking [3].

Herein, we report an investigation of Li-Si phase evolution under real-time solid-state chemical lithiation of single-crystalline $c$-Si membranes by probe-corrected scanning and transmission electron microscopy (S/TEM) coupled with electron energy-loss spectroscopy (EELS). After Li evaporation on to $35 \mathrm{~nm}$-thick windows $<100>$-oriented $p$-doped Si membranes were safely transferred to a microscope using a vacuum transfer TEM holder. Low-loss EEL spectra of such a membrane are dominated by plasmon peaks, which positions are directly related to valence electron densities $n$ as $E_{\mathrm{p}}=\hbar \omega_{\mathrm{p}}, \omega_{\mathrm{p}}=\left[n \mathrm{e}^{2} / \varepsilon_{0} \mathrm{~m}\right]^{0.5}$. No metallic Li was detected 30 minutes after $\mathrm{Li}$ evaporation indicating fast reactive lithiation kinetics. Using experimental and theoretical data from the Lorentz-Drude model and DFT calculations [1, 3], we have described relationships between $E_{\mathrm{p}}{ }^{2} \propto n$ vs. Li content $\mathrm{x}$ for various Li-Si intermetallic phases by an exponential decay function, $E_{\mathrm{p}}{ }^{2}=A_{1} \exp \left(-\mathrm{x} / t_{1}\right)+\mathrm{y}_{0}$, where least-squares-(lsq) fitted parameters $A_{1}=-$ $18.5 \pm 8.5, t_{1}=-38.5 \pm 6.1$, and $y_{0}=304.8 \pm 18.2$, respectively (Fig. 1a). Determining phase and chemical compositions and thickness from a Kramers-Kronig sum rule by HAADF STEM-EEL spectroscopic imaging, we have found a Li-rich metastable silicide glass with an atomic ratio of $\mathrm{Li} / \mathrm{Si} » 2.2$, close to a $\mathrm{Li}_{7} \mathrm{Si} 3$ that coexists with $c$-Si (Figs. $1 \mathrm{~b}$ and 2). The Lorentz-Drude models for $c$-Si (Fig. 1b, red curve) and metastable $m$-Li7 $\mathrm{Si}_{3}$ (Fig. 1b, green curve) were utilized for non-linear least-squares (NLLS) fitting of the spectra for each phase and total fit to derive the local phase distributions. In the absence of an external electric field, room temperature lithiation of $c-\mathrm{S}$ is driven by the Li concentration gradient and proceeds via Li-consuming fast silicidation reaction which involves diffusive solid-state amorphization (Fig. 2). With an increasing extent of lithiation together with transformations of the electronic band structure, the $\mathrm{Si}-\mathrm{Si}$ bonds in the diamond $c$-Si structure are successively broken, resulting in the sequential formation of ordered 1 to $2 \mathrm{~nm}$ five-membered or four-membered Si clusters distributed in the Si matrix distorted by mechanical stresses. However, room temperature recrystallization of equilibrium intermetallic Li-Si phases appeared frustrated, and after the initial fast reaction step, the metastable silicide glass did not further evolve towards the Li-richest $\mathrm{Li}_{15} \mathrm{Si} 4$ phase. The obtained results are compared with liquid phase electrochemical lithiation of $c-\mathrm{Si}$ in a $\mathrm{LiClO}_{4}$-dimethyl carbonate electrolyte when a solid electrolyte interface is present [1] and direct injection of $\mathrm{Li}^{+}$ions into $c$-Si using focused $\mathrm{Li}^{+}$ion beams [4]. 

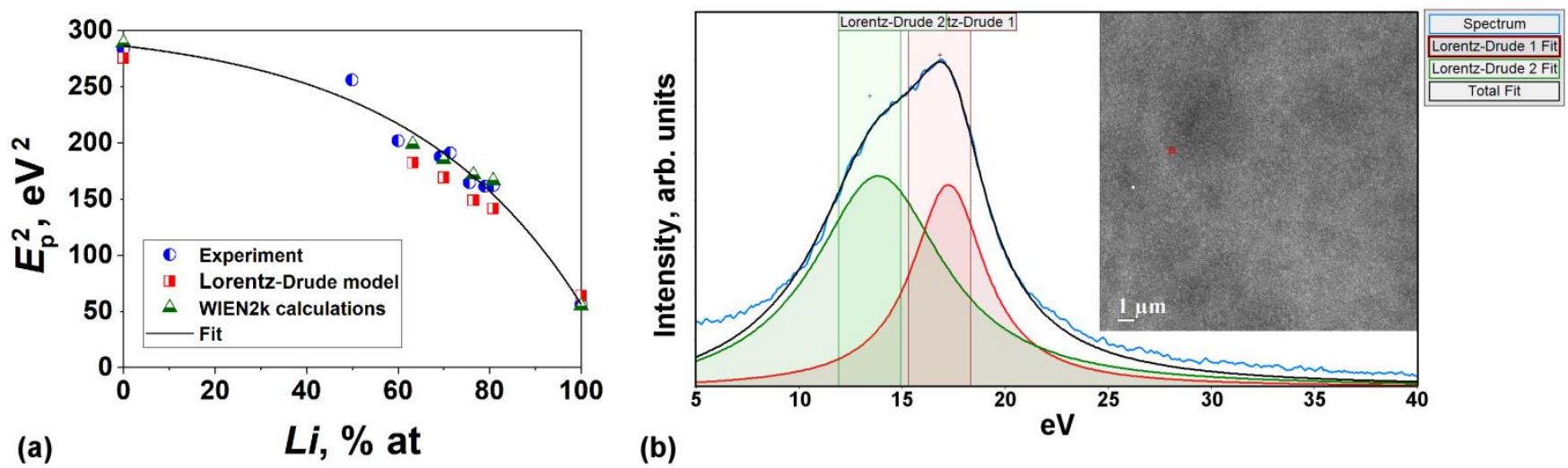

Figure 1. Figure 1. (a) Experimental and calculated values of Ep2 for different Li-Si alloys vs. Li content lsq-fitted with an exponential decay function $\mathrm{Ep} 2=\mathrm{A} 1 \exp (-\mathrm{x} / \mathrm{t} 1)+\mathrm{y} 0, \mathrm{~A} 1=-18.5 \pm 8.5, \mathrm{t} 1=-38.5 \pm 6.1$, y0=304.8 \pm 18.2 . (b) Low-loss EEL spectrum (a single scattering distribution, blue) acquired from the area marked by the red box in (b) shows plasmon peaks at $13.7 \mathrm{eV}$ assigned to a Li7Si3 glass and the bulk $\mathrm{Si}$ plasmon at $16.8 \mathrm{eV}$. Two Lorentz-Drude models (red and green curves) used for NLLS fitting of the spectrum for Li7Si3 and c-Si, respectively, and the total fit (black curve) are shown. HAADF STEM (inset) demonstrates random mass-thickness variations over a lithiated membrane.

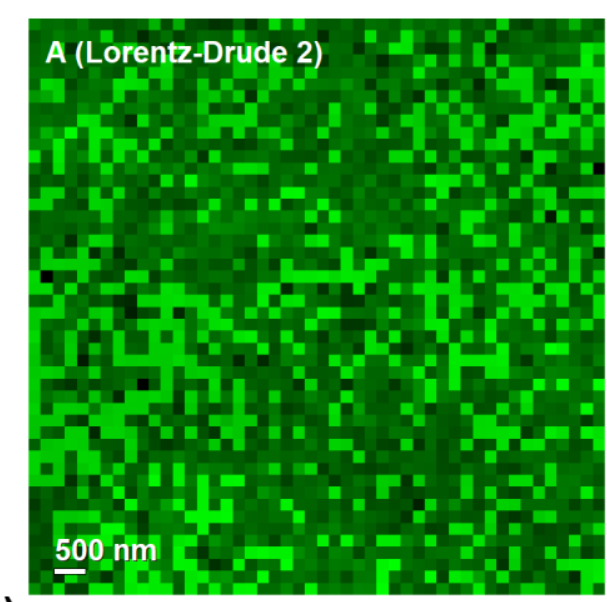

(a)

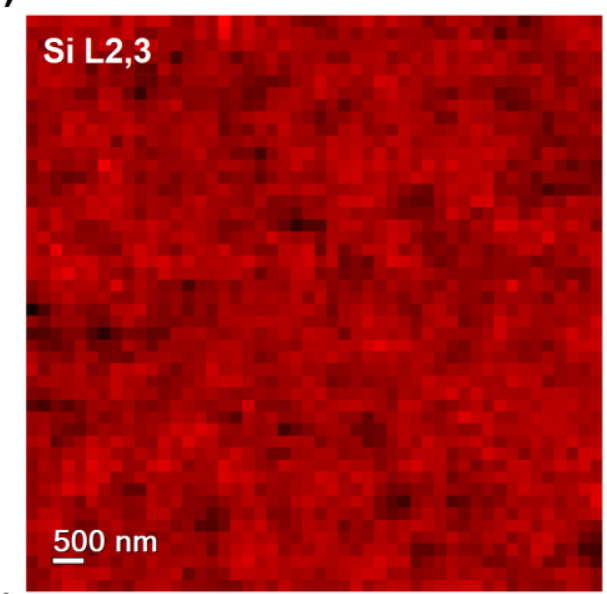

(c)

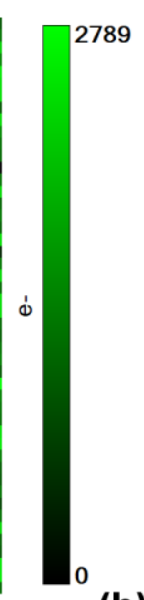

(b)
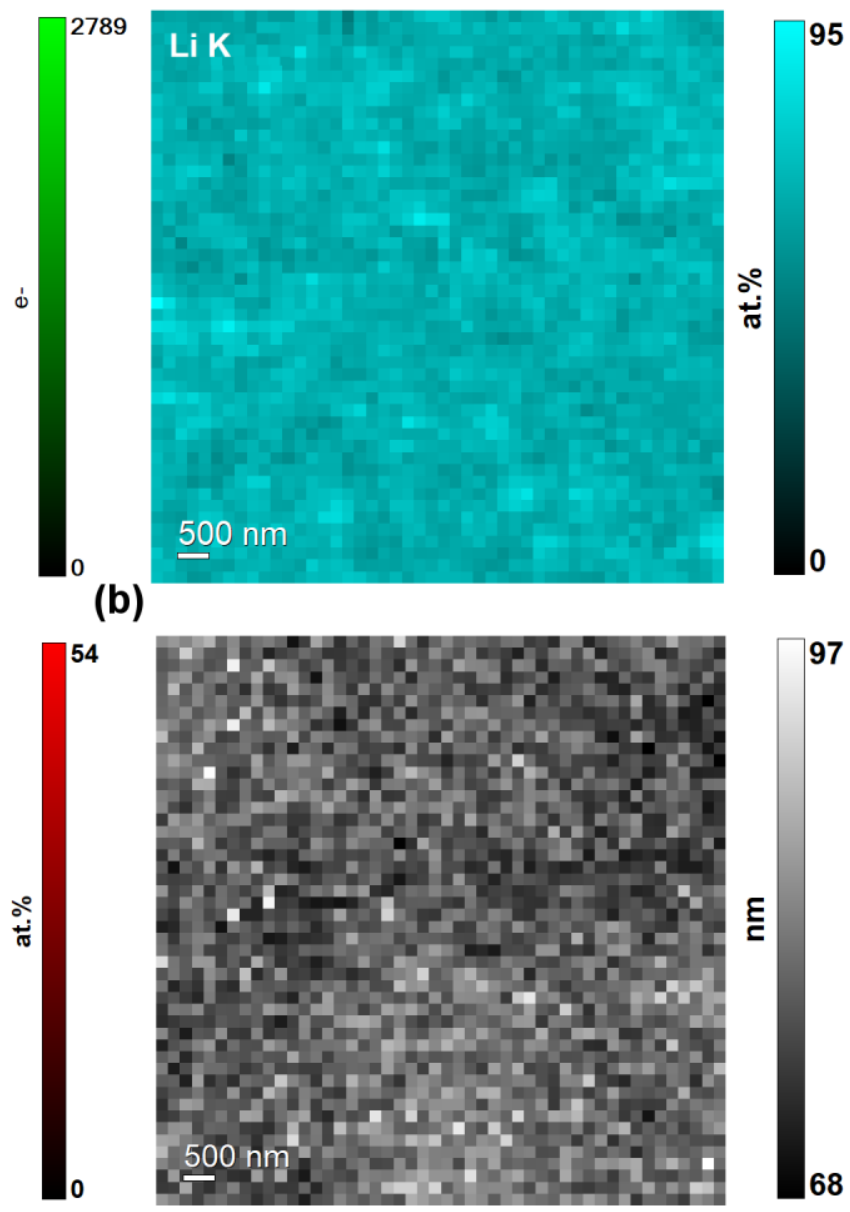

(d) 
Figure 2. Figure 2. STEM-EELS SI of a lithiated Si window area: (a) Li7Si3 phase distribution derived by NLLS fitting according to a procedure shown in Figlb; (b) quantitative Li K map; (c) quantitative Si L2,3 map; (d) absolute thickness map derived using a Kramers-Kronig sum rule and refractive index of $\mathrm{Si}, \mathrm{n}=3.98, \mathrm{t} / \lambda$ varied from 0.3 up to 1.1 .

\section{References}

[1] VP Oleshko, et al, Microsc. Microanal. 24 (S1), (2018) 1480; ibid 26 (S2) (2020) 1448.

[2] Choi JW, Aurbach D (2016) Nature Rev 1,1-16.

[3] Boniface, M., et al (2016) Nano Lett. 16, 7381-7388.

[4] McGehee W.A., et al, ACS Nano (2019) 13, 8012-8022 